\title{
Multi-gram-scale synthesis of a versatile syn-anti stereotriad by a short and cost-effective route
}

\author{
David J. Galler, Gabriela C. Bermudez, and Kathlyn A. Parker* \\ Department of Chemistry, State University of New York at Stony Brook, Stony Brook, \\ New York 11794, USA \\ Email: Kathlyn.parker@stonybrook.edu
}

Dedicated to Professor George A. Kraus in tribute to his many contributions to synthetic methodology and total synthesis

Received 02-05-2019

Accepted 03-22-2019

Published on line 04-24-2019

\section{Abstract}

The polyketide family of natural products includes numerous biologically important molecules that exhibit a variety of complex structures. The biosynthesis of these diverse structures relies on the iterative assembly of individual segments controlled by the enzymes of the polyketide synthase (PKS) family. In the synthesis laboratory, access to stereospecifically-prepared ketide building blocks can be both challenging and costlimiting. We report an efficient, multigram-scale synthesis of the syn-anti synthon $(2 S, 3 R, 4 S)(E)-6$-cyclohexyl-3[(4-methoxybenzyl)oxy]-2,4-dimethylhex-5-en-1-ol from the commercially available cyclohexanecarboxaldehyde in excellent overall yield and purity.<smiles>CC1CCCCC1</smiles>

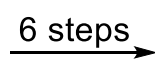<smiles>CCCCCCO[C@@H](CO)[C@@H](C)/C=C/C1CCCCC1</smiles>

$54 \%, 95 \%$ ee

Keywords: Polyketides, polypropionates, enantioselection, synthon, catalysis 


\section{Introduction}

The polyketides are complex and diverse natural products. ${ }^{1,2}$ Many are in drug trials ${ }^{1}$ and clinical use. ${ }^{2}$ About $22 \%$ of prescription drugs that are derived from natural products contain polyketide units. ${ }^{3}$ The large number of asymmetric centers in most of these substances, often in contiguous runs, pose major obstacles to their synthetic construction.

The asymmetric synthesis of polyketide compounds is performed in nature by polyketide synthetases (PKSs) which direct the necessary aldol couplings, reductions, and, in some cases, dehydrations. ${ }^{4-6}$ An oftenused strategy in the laboratory synthesis of polyketides is the iterative coupling of small chiral building blocks. ${ }^{6}$ Such syntheses, which have largely focused on stereospecificity, can be costly on a large scale. ${ }^{7,8}$ For example, the practical total synthesis of discodermolide (Figure 1), a marine polyketide, remains a formidable challenge. We describe the multi-gram, catalytic, asymmetric preparation of stereotriad (1a) by a six-step route that offers convenience and promises further scalability.

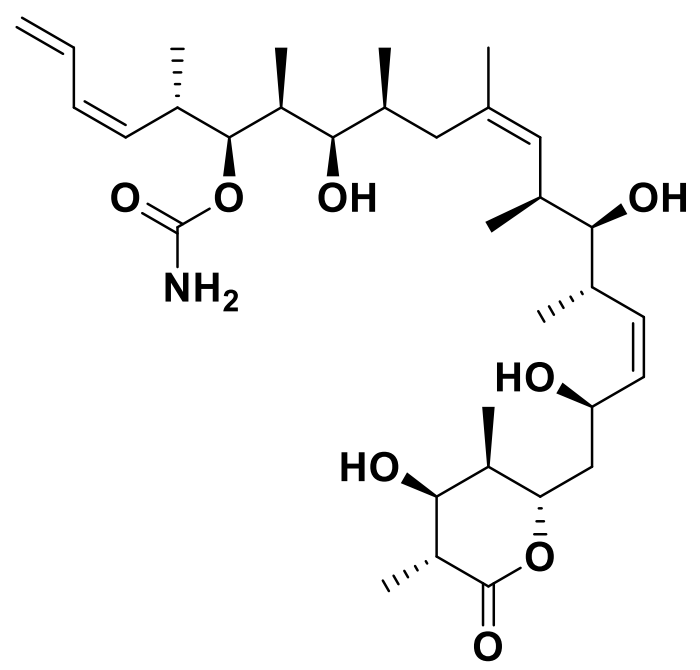

Figure 1. The natural polyketide (+)-discodermolide.

In earlier work on an approach to discodermolide, we designed the chiral syn-anti stereotriad (1) and reported a small-scale, five-step, catalytic asymmetric preparation of the syn-anti stereotriad building blocks (1b) and (1c)..$^{9-11}$ These synthons and their equivalents offer particularly attractive options for extending the polyketide chain in both directions. Of special note is the potential for ozonolysis as a method of liberating an aldehyde at one terminus.

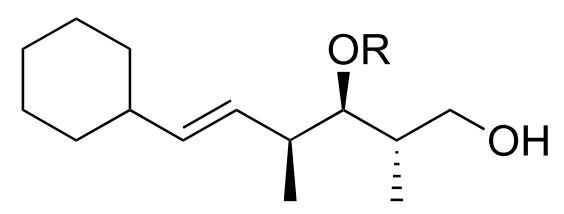

$1 \mathrm{a}: \mathrm{R}=\mathrm{PMB}$

$1 \mathrm{~b}: \mathrm{R}=\mathrm{TES}$

1c: $R=M O M$

To date, the syn-anti stereotriad unit (2) from Leighton, ${ }^{12,13}$ (3) from Smith, ${ }^{14,15}$ and the identical NovartisSmith-Paterson ${ }^{16-20}$ unit are the only examples of syn-anti building blocks that have been prepared on multi- 
gram scales. Each of these substances is easily modifiable at each terminus for ease of continued synthetic elaboration.<smiles>C=C[C@H](C)[C@H](O)[C@@H](C)C(C)=O</smiles>

2<smiles>COc1ccc(COC[C@@H](C)[C@H](O)[C@H](C)C(=O)N(C)OC)cc1</smiles>

3

We report the large-scale synthesis of synthon 1a using Carreira's asymmetric addition reaction ${ }^{21-24}$ as a key step in an expansion of our previous approach. This preparation is outlined in Scheme 1.
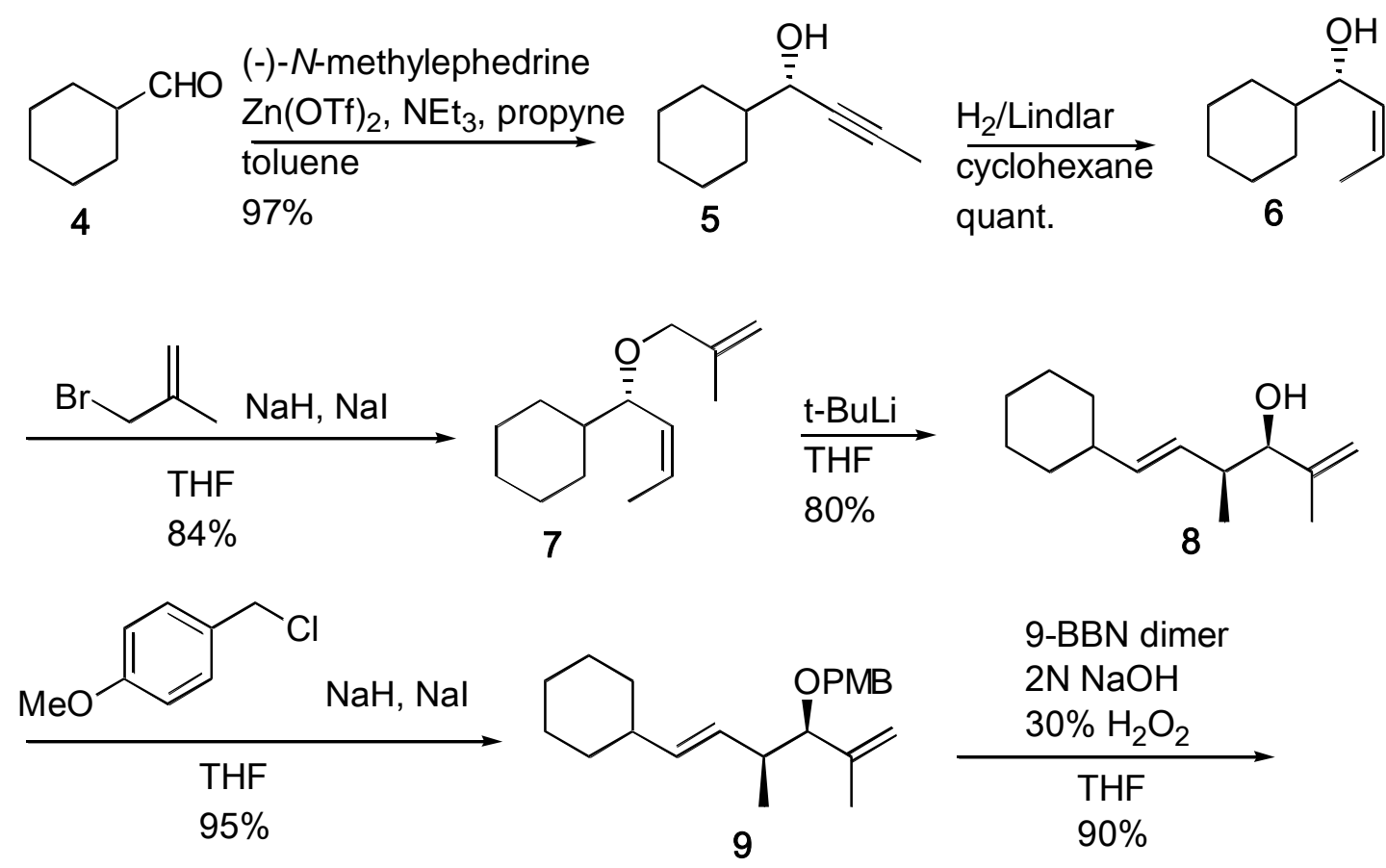<smiles>[Y10]O[C@H]([C@@H](C)/C=C/C1CCCCC1)[C@@H](C)CO</smiles>

Scheme 1. The synthesis of synthon 1a (54\% overall yield, $95 \%$ ee).

\section{Results and Discussion}

Our previously reported approach to stereotriad (1) was modified to improve the yield and ease of handling. In the earlier work, ${ }^{9-11}(-)-N$-methylephedrine was used as a chiral auxiliary to direct a propenylation of cyclohexanecarboxyaldehyde (4) to produce the alcohol (6) in one step. These reaction conditions proved to be highly moisture sensitive, difficult to maintain in large batches, and challenging to reproduce. This step was 
replaced by a two-step procedure: Carreira propynylation and hydrogenation. The Carreira reaction ${ }^{21-24}$ afforded the alcohol (5) in $97 \%$ yield with $95 \%$ ee.

We note, however, that zinc triflate is an expensive source of zinc(II) ion. Thus, other zinc salts were considered to reduce the cost of this step while retaining yield and ee. These results (Table 1) highlight the clear advantage of zinc triflate over zinc bromide and zinc chloride. Despite the variation in overall yield depending on the zinc salt, the overall ee of the product remained unaffected. Although we were unable to find an acceptable alternative to zinc triflate, we were able to reduce the amount of salt to 10 mol \% without sacrificing yield using extended reaction times. (-)-N-Methylephedrine can be recovered, quantitatively, from aqueous extraction. ${ }^{25}$

Table 1. Summary of the optimization of Carreira's conditions ${ }^{\mathrm{a}}$.

\begin{tabular}{cccc}
\hline Salt & Mol \% & Reaction time & Yield (\%) \\
\hline Zinc(II) chloride & 100 & $48 \mathrm{~h}$ & 36 \\
Zinc(II) bromide & 100 & $48 \mathrm{~h}$ & 45 \\
Zinc(II) triflate & 100 & $48 \mathrm{~h}$ & 96 \\
Zinc(II) triflate & 60 & $48 \mathrm{~h}$ & 97 \\
Zinc(II) triflate & 10 & $7 \mathrm{~d}$ & 94 \\
\hline
\end{tabular}

a All reactions were performed with toluene as the solvent. The ee, as determined by Mosher ester analysis, was unaffected by the $\mathrm{Zn}(\mathrm{II})$ source.

The subsequent reduction of alcohol (5) with Lindlar's catalyst provided the necessary (Z)-olefinic alcohol (6) in quantitative yield. Formation of ether (7) was performed under Finkelstein conditions. ${ }^{26}$ Our synthetic intermediate syn diad (8) was easily isolated after a [2,3]-Wittig rearrangement of 7.27-33 Optimum conditions for this rearrangement required the use of excess $t$-BuLi (Table 2). This protocol afforded the rearranged 8 without side products. ${ }^{33}$ Unreacted starting material was easily recovered by chromatography. 
Table 2. Optimization of the [2,3]-Wittig rearrangement ${ }^{\mathrm{a}}$.

\begin{tabular}{ccc}
\hline Base & Temperature & Yield (\%) \\
\hline$n$-BuLi, $t$-BuOK & $-78{ }^{\circ} \mathrm{C}$ to $0{ }^{\circ} \mathrm{C}$ & 35 \\
$n$-BuLi, $t$-BuOK, HMPA & $-78{ }^{\circ} \mathrm{C}$ to $0{ }^{\circ} \mathrm{C}$ & 0 \\
$n$-BuLi, LDA & $-78{ }^{\circ} \mathrm{C}$ to $0{ }^{\circ} \mathrm{C}$ & 0 \\
$n$-BuLi, $t$-BuOK & $-78{ }^{\circ} \mathrm{C}$ to $0{ }^{\circ} \mathrm{C}$, then $0{ }^{\circ} \mathrm{C}$ to $-78{ }^{\circ} \mathrm{C}$ & 66 \\
$\mathrm{n}$-BuLi & $-78{ }^{\circ} \mathrm{C}$ to $0{ }^{\circ} \mathrm{C}$ & 50 \\
$s e c$-BuLi & $-78{ }^{\circ} \mathrm{C}$ to $0{ }^{\circ} \mathrm{C}$ & 55 \\
$t$-BuLi & $-78{ }^{\circ} \mathrm{C}$ to $0{ }^{\circ} \mathrm{C}$ & 60 \\
$t$-BuLib & $-90{ }^{\circ} \mathrm{C}$ to $-20{ }^{\circ} \mathrm{C}$ & 80 \\
\hline
\end{tabular}

a.All reactions were performed with $100 \mathrm{mg}$ of substrate (7) in THF under argon atmosphere for $4 \mathrm{~h}$. b. No byproduct was observed. " Yields reflect product isolated following chromatography.

Finally, alcohol (8) was converted to the desired syn-anti synthon (1a) by a two-step procedure, i.e., protection with $\mathrm{p}$-methoxybenzyl chloride (PMB-Cl) followed by selective oxidation of the terminal olefin with crystalline 9-borabicyclo[3.3.1]nonane (9-BBN).

We imagine that synthon (1a) may be incorporated into large polyketides through easily performed transformations. ${ }^{34-40}$

\section{Conclusions}

In summary, we have successfully completed a multi-gram asymmetric synthesis of the syn-anti synthon (1a) in six steps in $54 \%$ overall yield with $95 \%$ ee. We envision this stereotriad to be a precursor to a variety of synthetically useful compounds that contain contiguous stereochemical centers. We believe that the cost effectiveness of this approach is advantageous for large-scale polyketide synthesis.

\section{Experimental Section}

General. Solvents were dried over calcium hydride. Thin-layer chromatography was performed on Agela plates, $0.25 \mathrm{~mm}$ thickness, $60 \AA$ F254. Plates were stained with $15-20 \%$ phosphomolybdic acid (PMA) or visualized by UV (254 nm). Commercially-available reagents were purchased from Alfa Aesar. All NMR spectra were recorded on Bruker $500 \mathrm{MHz}$ and $700 \mathrm{MHz}$ spectrometers. NMR solvents were purchased from Cambridge Isotope Laboratories (Tewksbury, Massachusetts, USA). High-resolution mass spectra (HRMS) were acquired with electrospray ionization (positive mode) at the Stony Brook University Mass Spectrometry Lab on an Agilent LC-UV-TOF model G6224A oaTOF. IR spectra were collected on a Thermo Scientific Nicolet iS10 FTIR spectrophotometer. 
(S)-1-Cyclohexylbut-2-yn-1-ol (5). ${ }^{23}$ Triethylamine (25.0 g, $\left.246 \mathrm{mmol}\right), \mathrm{Zn}(\mathrm{OTf})_{2}$ (39.0 g, $\left.107 \mathrm{mmol}\right)$, and (-)- $\mathrm{N}$ methylephedrine $(21.0 \mathrm{~g}, 117 \mathrm{mmol})$ were added to an argon-filled $2 \mathrm{~L}$ pressure vessel along with $1.2 \mathrm{~L}$ of dry toluene. The mixture was stirred for $2 \mathrm{~h}$ at room temperature after which cyclohexanecarboxyaldehyde (20.3 $\mathrm{g}, 181 \mathrm{mmol}$ ) was added. The vessel was chilled to $-78{ }^{\circ} \mathrm{C}$ in an acetone/dry ice bath. Propyne gas (9.8 g, 245 $\mathrm{mmol}$ ) was added and the vessel was sealed with a Teflon screw cap. The reaction mixture was warmed to room temperature and stirred for $72 \mathrm{~h}$. The reaction was quenched with a saturated solution of $\mathrm{NH}_{4} \mathrm{Cl}$. The organic phase was washed three times with $500 \mathrm{~mL}$ saturated $\mathrm{NH}_{4} \mathrm{Cl}$ solution, dried over $\mathrm{MgSO}_{4}$, and concentrated under reduced pressure to afford a clear, colorless oil. Yield: $26.6 \mathrm{~g}$ (97.0\%).

(S)(Z)-1-Cyclohexylbut-2-en-1-ol (6). ${ }^{9}$ Alcohol 5 (20.0 g, $131 \mathrm{mmol}$ ) was dissolved in $200 \mathrm{~mL}$ of dry cyclohexane and added to a $500 \mathrm{~mL}$ Parr shaker flask. Lindlar's catalyst $(1.67 \mathrm{~g}, 0.790 \mathrm{mmol})$ was added. The vessel was then flushed with hydrogen and pressurized to 52.0 psi four times until the hydrogen uptake ceased. The mixture was then filtered through Celite and washed through with cyclohexane. The filtrate was concentrated under reduced pressure to afford a pale yellow oil. Yield: $22 \mathrm{~g}$ (quantitative).

(S)(Z)-\{1-[(2-Methylallyl)oxy]but-2-en-1-yl\}cyclohexane (7). ${ }^{9}$ Dry THF (300 mL) was added to a $500 \mathrm{~mL}$ argonflushed two-neck round-bottom flask which was cooled in an ice bath. Sodium hydride $(60 \%, 29.0 \mathrm{~g}, 720$ mmol) was slowly added into the reaction vessel and the reaction mixture was stirred for 15 minutes. 3-Bromo-2-methylpropene (32.4 g, $240 \mathrm{mmol}$ ), was then added, followed by the dropwise addition of alcohol (6) $(22.0 \mathrm{~g}, 143 \mathrm{mmol})$ in THF $(10 \mathrm{~mL})$. The reaction mixture was stirred for an additional 10 minutes then sodium iodide $(21.4 \mathrm{~g}, 143 \mathrm{mmol})$ was added. The solution was warmed to room temperature and left overnight. The mixture was chilled in an ice bath and quenched with the slow addition of water until two layers formed. The aqueous phase was then extracted three times with ether (300 $\mathrm{mL}$ portions). The combined organic-phase solution was dried over $\mathrm{MgSO}_{4}$ and concentrated under reduced pressure. The crude material was purified using column chromatography (silica gel, 20:1 hexanes:ethyl acetate) to afford a pale yellow oil. Yield: $25.3 \mathrm{~g}(84 \%)$.

$(3 R, 4 S)(E)-6-C y c l o h e x y l-2,4-d i m e t h y l h e x a-1,5-d i e n-3-o l ~(8) .{ }^{9}$ A flame-dried $500 \mathrm{~mL}$ three-neck round-bottom flask was purged with argon and cooled to $-90{ }^{\circ} \mathrm{C}$. tert-Butyllithium $(100 \mathrm{~mL}, 160 \mathrm{mmol})$ was transferred into an argon-purged addition funnel via cannula, then, slowly dripped into the reaction vessel over 20 minutes. Ether (7) $(11.0 \mathrm{~g}, 53.0 \mathrm{mmol})$ in dry THF $(10 \mathrm{~mL})$ was then added dropwise to the reaction vessel followed by 12-crown-4 $(0.26 \mathrm{~mL})$ and the mixture was stirred for $4 \mathrm{~h}$ at $-90^{\circ} \mathrm{C}$. The reaction was quenched by slow dropwise addition of water and warmed to room temperature over $2 \mathrm{~h}$. The aqueous phase was extracted three times with ethyl acetate (100 mL portions). The combined organic solution was dried over anhydrous $\mathrm{MgSO}_{4}$ and concentrated under reduced pressure. The crude material was purified by column chromatography (silica gel 20:1 hexanes:ethyl acetate) to afford product as a yellow oil. Yield: $8.55 \mathrm{~g}$ (78\%).

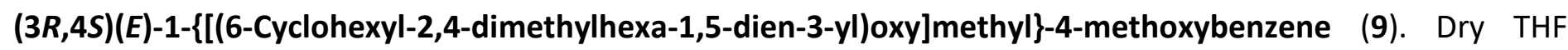
$(100 \mathrm{~mL}$ ) was added to a $250 \mathrm{~mL}$ argon-flushed two-neck round-bottom flask and cooled in an ice bath. Sodium hydride $(60 \%, 1.72 \mathrm{~g}, 72.0 \mathrm{mmol})$ was slowly added to the reaction vessel and the resulting suspension was stirred for 15 minutes. Alcohol (8) $(3.00 \mathrm{~g}, 14.4 \mathrm{mmol})$ was dissolved in dry THF (15 mL) and added into the reaction vessel followed by para-methoxybenzyl chloride $(5.88 \mathrm{~mL}, 43.2 \mathrm{mmol})$. After 10 minutes of stirring, sodium iodide $(2.15 \mathrm{~g}, 14.4 \mathrm{mmol})$ was added and the reaction mixture was brought to room temperature and left for $72 \mathrm{~h}$. The reaction mixture was chilled in an ice bath and quenched by the slow addition of water until two layers formed. The aqueous phase was extracted three times with ether $(50 \mathrm{~mL}$ portions). The combined organic solution was dried over $\mathrm{MgSO}_{4}$ and concentrated under reduced pressure. The crude material was purified by column chromatography (silica gel, 20:1 hexane:ethyl acetate) to afford the product as a pale yellow oil. Yield: $4.50 \mathrm{~g}$ (95.0\%). $\mathrm{R}_{f} 0.66$ (20:1 hexane : ethyl acetate) ${ }^{1} \mathrm{H}-\mathrm{NMR}\left(\mathrm{CDCl}_{3}\right.$, 
$500 \mathrm{MHz}): \delta 7.25(\mathrm{~d}, J 8 \mathrm{~Hz}, 2 \mathrm{H}), 6.87(\mathrm{~d}, J 8 \mathrm{~Hz}, 2 \mathrm{H}), 5.30(\mathrm{~m}, 1 \mathrm{H}), 5.12(\mathrm{~m}, 1 \mathrm{H}), 4.94(\mathrm{~s}, 1 \mathrm{H}), 4.82(\mathrm{~s}, 1 \mathrm{H}), 4.44$ $(\mathrm{d}, J 11 \mathrm{~Hz}, 1 \mathrm{H}), 4.15(\mathrm{~d}, J 11 \mathrm{~Hz}, 1 \mathrm{H}), 3.80(\mathrm{~s}, 3 \mathrm{H}), 3.34(\mathrm{~d}, J 9 \mathrm{~Hz}, 1 \mathrm{H}), 2.28(\mathrm{~m}, 1 \mathrm{H}), 1.84(\mathrm{~m}, 1 \mathrm{H}), 1.64(\mathrm{~m}, 8 \mathrm{H})$, $1.19(\mathrm{~m}, 3 \mathrm{H}), 1.05$ (d, J $7 \mathrm{~Hz}, 3 \mathrm{H}), 0.99(\mathrm{~m}, 2 \mathrm{H}) .{ }^{13} \mathrm{C}-\mathrm{NMR}\left(\mathrm{CDCl}_{3}, 125 \mathrm{MHz}\right): \delta$ 159.0, 143.7, 135.7, 131.0, 129.6, 129.4, 129.3, 114.6, 114.7, 113.7, 113.5, 87.7, 69.7, 55.3, 40.6, 39.5, 33.11, 33.08, 26.2, 26.1, 17.5, 17.1. IR cm 1 ( $\mathrm{NaCl}$ ): 3069, 2923, 2850, 1650, 1613, 1586, 1513, 1449, 1370, 1348, 1301, 1247, 1207, 1172, 1109, 1073, 1039, 1011, 967, 899, 844, 821, 756, 637. HRMS (ESI $\left.{ }^{+}\right): \mathrm{C}_{22} \mathrm{H}_{32} \mathrm{O}_{2} 328.2402$, calc for $(\mathrm{M}+\mathrm{H})$ : 329.2475, found 329.2474.

$(2 S, 3 R, 4 S)(E)-6-C y c l o h e x y l-3-[(4-m e t h o x y b e n z y l) o x y]-2,4-d i m e t h y l h e x-5-e n-1-o l \quad(1 a)$. In an argon-filled round-bottom flask was added dry THF $(25 \mathrm{ml})$ and ether $(9)(1.00 \mathrm{~g}, 3.03 \mathrm{mmol})$. The solution was cooled to -5 ${ }^{\circ} \mathrm{C}$, and 9-borabicyclo[3.3.1]nonane (9-BBN) dimer (1.48 g, $\left.6.06 \mathrm{mmol}\right)$ in THF $(5 \mathrm{~mL})$ was added dropwise. The reaction mixture was warmed to room temperature and tracked by TLC. The reaction was complete after $12 \mathrm{~h}$, and the mixture was cooled back to $-5{ }^{\circ} \mathrm{C}$. Over 20 minutes, $2 \mathrm{~N} \mathrm{NaOH}(18 \mathrm{~mL}, 36 \mathrm{mmol})$ was added followed by $30 \% \mathrm{H}_{2} \mathrm{O}_{2}(18 \mathrm{~mL})$. The reaction mixture was warmed to room temperature and left to stir for $5 \mathrm{~h}$, then diluted with ether $(50 \mathrm{~mL})$ and washed three times with sat. $\mathrm{NH}_{4} \mathrm{Cl}(25 \mathrm{~mL}$ portions). The organic solution was dried over $\mathrm{MgSO}_{4}$, filtered, and concentrated under reduced pressure. The crude oil was purified by column chromatography (silica gel, 10:1 hexane:ethyl acetate) to afford a clear colorless oil. Yield: $954 \mathrm{mg}(90 \%) . \mathrm{R}_{f}$ 0.15 (10:1 hexane:ethyl acetate) ${ }^{1} \mathrm{H}-\mathrm{NMR}\left(\mathrm{CDCl}_{3}, 500 \mathrm{MHz}\right): \delta 7.28(\mathrm{~d}, J 8 \mathrm{~Hz}, 2 \mathrm{H}), 6.89(\mathrm{~d}, J 8 \mathrm{~Hz}, 2 \mathrm{H}), 5.42(\mathrm{~m}$, $2 \mathrm{H}), 4.61(\mathrm{~d}, J 11 \mathrm{~Hz}, 1 \mathrm{H}), 4.48(\mathrm{~d}, J 11 \mathrm{~Hz}, 1 \mathrm{H}), 3.81(\mathrm{~s}, 3 \mathrm{H}), 3.72(\mathrm{dd}, J 8 \mathrm{~Hz}, 3 \mathrm{~Hz}, 1 \mathrm{H}), 3.56(\mathrm{~d}, J 6 \mathrm{~Hz}, 1 \mathrm{H}), 3.26$ $(\mathrm{t}, J 6 \mathrm{~Hz}, 1 \mathrm{H}), 2.82(\mathrm{~s}, 1 \mathrm{H}), 2.47(\mathrm{~m}, 1 \mathrm{H}), 1.92(\mathrm{~m}, 3 \mathrm{H}), 1.71(\mathrm{~m}, 6 \mathrm{H}), 1.52(\mathrm{~m}, 3 \mathrm{H}), 1.29(\mathrm{~m}, 2 \mathrm{H}), 1.17,(\mathrm{tt}, J 12$ $\mathrm{Hz}, 3 \mathrm{~Hz}, 1 \mathrm{H}), 1.09$ (d, J $7 \mathrm{~Hz}, 3 \mathrm{H}), 1.06(\mathrm{~m}, 1 \mathrm{H}), 1.00(\mathrm{~d}, J 7 \mathrm{~Hz}, 3 \mathrm{H}){ }^{13} \mathrm{C}-\mathrm{NMR}\left(\mathrm{CDCl}_{3}, 125 \mathrm{MHz}\right): \delta$ 159.2, 136.1, $130.9,129.4,88.9,74.7,66.0,55.2,40.7,39.8,37.2,34.7,33.1,33.0,27.4,26.1,26.0,22.6,15.5 . \mathrm{IR} \mathrm{cm}^{-1}$ (NaCl): 3418, 2919, 2236, 2061, 1878, 1739, 1613, 1586, 1514, 1448, 1348, 1301, 1247, 1173, 1036, 975, 892, 822, 757, 733. HRMS $\left(E S I^{+}\right): \mathrm{C}_{22} \mathrm{H}_{34} \mathrm{O}_{3} 346.2508$, calc for $(\mathrm{M}+\mathrm{H}): 347.2581$, found 347.2583

\section{Acknowledgements}

D.J.G. and G.C.B. were Fellows of the Graduate Assistance in Areas of National Need (GAANN) Program of the US Department of Education, P200A100044 and P20A120094. We thank Professor Francis Johnson (Stony Brook University) for his continued mentorship during this project. We extend thanks to Francis Picart, Fang Liu, and James Marecek from the NMR Facilities at Stony Brook, and Béla Ruzsicska from ICB\&DD Mass Spectrometry Laboratory for facilitating instrumental setup and data acquisition.

\section{Supplementary Material}

Supplementary data associated with this article can be found in the online version.

\section{References}

1. Lorente, A.; Makowski, K.; Albericio, F.; Álvarez, M. Ann. Mar. Biol. Res. 2014, 1, 1003-1013.

2. Newman, D. J.; Cragg, G. M. J. Nat. Prod. 2012, 75, 311-335.

https://doi.org/10.1021/np200906s 
3. WHO. In WHO Model List of Essential Medicines, 2015; 19th Ed, pp 28-31.

4. McDaniel, R.; Welch, M.; Hutchinson, C. R. Chem. Rev. 2005, 105, 543-558. https://doi.org/10.1021/cr0301189

5. Hill, A. M. Nat. Prod. Rep. 2006, 23, 256-320. https://doi.org/10.1039/B301028G

6. Yeung, K. S.; Paterson, I. Chem. Rev. 2005, 105, 4237-4313. https://doi.org/10.1021/cr040614c

7. Nicolaou, K. C.; Nold, A. L.; Milburn, R. R.; Schindler, C. S. Angew. Chem. Int. Ed. 2006, 45, 6527-6532. https://doi.org/10.1002/anie.200601867

8. Shang, S.; Iwadare, H.; Macks, D. E.; Ambrosini, L. M.; Tan, D. S. Org. Lett. 2007, 9, 1895-1898. https://doi.org/10.1021/ol070405p

9. Parker, K. A.; Cao, H. Org. Lett. 2006, 8, 3541-3544. https://doi.org/10.1021/ol0612612

10. Parker, K. A.; Wang, P. Org. Lett. 2007, 9, 4793-4796. https://doi.org/10.1021/ol702144u

11. Cao, H.; Parker, K. A. Org. Lett. 2008, 10, 1353-1356. https://doi.org/10.1021/ol7029933

12. Foley, C. N.; Leighton, J. L. Org. Lett. 2014, 16, 1180-1183. https://doi.org/10.1021/ol500051e

13. Foley, C. N.; Leighton, J. L. Org. Lett. 2015, 17, 5858-5861. https://doi.org/10.1021/acs.orglett.5b03034

14. Smith, A. B.; Beauchamp, T. J.; Lamarche, M. J.; Kaufman, M. D.; Qiu, Y.; Arimoto, H.; Jones, D. R.; Kobayashi, K. J. Am. Chem. Soc. 2000, 122, 8654-8664. https://doi.org/10.1021/ja0015287

15. Smith, A. B.; Kaufman, M. D.; Beauchamp, T. J.; LaMarche, M. J.; Arimoto, H. Org. Lett. 1999, 1, $1823-1826$. https://doi.org/10.1021/ol9910870

16. Mickel, S. J.; Sedelmeier, G. H.; Niederer, D.; Daeffler, R.; Osmani, A.; Schreiner, K.; Seeger-Weibel, M.; Bérod, B.; Schaer, K.; Gamboni, R.; Chen S.; Chen, W.; Jagoe, C. T.; Kinder, F. R.; Loo, M.; Prasad, K.; Repic, O.; Chieh, W-C.; Wang, R-M.; Waykole, L.; Xu, D. D.; Xue, S., Org. Process Res. Dev. 2004, 8, 92-100. https://doi.org/10.1021/op034130e

17. Mickel, S. J.; Sedelmeier, G. H.; Niederer, D.; Schuerch, F.; Grimler, D.; Koch, G.; Daeffler, R.; Osmani, A.; Hirni, A.; Schaer, K.; Chen S.; Chen, W.; Jagoe, C. T.; Kinder, F. R.; Loo, M.; Prasad, K.; Repic, O.; Chieh, W-C.; Wang, R-M.; Waykole, L.; Xu, D. D.; Xue, S., Org. Process Res. Dev. 2004, 8, 101-106. https://doi.org/10.1021/op0341317

18. Mickel, S. J.; Sedelmeier, G. H.; Niederer, D.; Schuerch, F.; Seger, M.; Schreiner, K.; Daeffler, R.; Osmani, A.; Bixel, D.; Loiseleur, O.; Chen S.; Chen, W.; Jagoe, C. T.; Kinder, F. R.; Loo, M.; Prasad, K.; Repic, O.; Chieh, WC.; Wang, R-M.; Waykole, L.; Xu, D. D.; Xue, S., Org. Process Res. Dev. 2004, 8, 113-121. https://doi.org/10.1021/op034133r

19. Mickel, S. J.; Sedelmeier, G. H.; Niederer, D.; Schuerch, F.; Koch, G.; Kuesters, E.; Daeffler, R.; Osmani, A.; Seeger-Weibel, M.; Schmid, E.; Chen S.; Chen, W.; Jagoe, C. T.; Kinder, F. R.; Loo, M.; Prasad, K.; Repic, O.; Chieh, W-C.; Wang, R-M.; Waykole, L.; Xu, D. D.; Xue, S.,Org. Process Res. Dev. 2004, 8, 107-112. https://doi.org/10.1021/op034132z 
20. Mickel, S. J.; Niederer, D.; Daeffler, R.; Osmani, A.; Kuesters, E.; Schmid, E.; Schaer, K.; Gamboni, R.; Chen, W.; Loeser, E.; Chen S.; Chen, W.; Jagoe, C. T.; Kinder, F. R.; Loo, M.; Prasad, K.; Repic, O.; Chieh, W-C.; Wang, R-M.; Waykole, L.; Xu, D. D.; Xue, S., Org. Process Res. Dev. 2004, 8, 122-130. https://doi.org/10.1021/op034134j

21. Frantz, D. E.; Fässler, R.; Tomooka, C. S.; Carreira, E. M. Acc. Chem. Res. 2000, 33, 373-381. https://doi.org/10.1021/ar9900780

22. El-Sayed, E.; Anand, N. K.; Carreira, E. M. Org. Lett. 2001, 3, 3017-3019. https://doi.org/10.1021/ol016431j

23. Diez, R. S.; Adger, B.; Carreira, E. M. Tetrahedron 2002, 58, 8341-8344. https://doi.org/10.1016/S0040-4020(02)00985-7

24. Sasaki, H.; Boyall, D.; Carreira, E. M. Helv. Chim. Acta 2001, 84, 964-971. https://doi.org/10.1002/1522-2675(20010418)84:4<964::AID-HLCA964>3.0.CO;2-I

25. Parker, K. A.; Xie, Q. Org. Lett. 2008, 10, 1349-1352. https://doi.org/10.1021/ol702989g

26. Streitwieser, A. Chem. Rev. 1956, 56, 571-752. https://doi.org/10.1021/cr50010a001

27. Nakal, T.; Mikaml, K. Org. React. 1994, Vol. 107, pp 105-209. https://doi.org/10.1002/0471264180.or046.02

28. Mikami, K.; Fujimoto, K.; Kasuga, T.; Nakai, T. Tetrahedron Lett. 1984, 25, 6011-6014. https://doi.org/10.1016/S0040-4039(01)81746-9

29. Thomasi, S. S.; Ladeira, C.; Ferreira, D.; da Fontoura Sprenger, R.; Badino, A. C.; Ferreira, A. G.; Venâncio, T. Helv. Chim. Acta 2016, 99, 281-285. https://doi.org/10.1002/hlca.201500038

30. Enders, D.; Backhaus, D.; Runsink, J. Angew. Chemie Int. Ed. English. 1994, 33 , 2098-2100. https://doi.org/10.1002/anie.199420981

31. Manabe, S. Chem. Commun. 1997, 737-738. https://doi.org/10.1039/a700906b

32. McGowan, G. Aust. J. Chem. 2002, 55, 799. https://doi.org/10.1071/CH02184

33. Tsai, D. J.; Midland M. M.; J. Am. Chem. Soc. 1985, 107, 3915-3918. https://doi.org/10.1021/ja00299a026

34. Yu, L.; Trujillo, M. E.; Miyanaga, S.; Saiki, I.; Igarashi, Y. J. Nat. Prod. 2014, 77, 976-982. https://doi.org/10.1021/np401071x

35. Dias, L. C.; Gonçalves, C. C. S. J. Braz. Chem. Soc. 2010, 21, 2012-2016. https://doi.org/10.1590/S0103-50532010001000030

36. Menche, D. Nat. Prod. Rep. 2008, 25, 905-918. https://doi.org/10.1039/b707989n

37. Riccio, R.; Bifulco, G.; Cimino, P.; Bassarello, C.; Gomez-Paloma, L. Pure Appl. Chem. 2003, 75, $295-308$. https://doi.org/10.1351/pac200375020295

38. Kretschmer, M.; Menche, D. Synlett 2010, 16, 2989-3007. https://doi.org/10.1055/s-0030-1259070

39. Fleury, E.; Lannou, M. I.; Bistri, O.; Sautel, F.; Massiot, G.; Pancrazi, A.; Ardisson, J. J. Org. Chem. 2009, 74, 7034-7045. https://doi.org/10.1021/j09012833 
40. Okada, Y.; Matsunaga, S.; Van Soest, R. W. M.; Fusetani, N. Org. Lett. 2002, 4, 3039-3042. https://doi.org/10.1021/ol0262791 